\title{
Health Technology Assessment: Wer hat in der Medizin das Sagen?
}

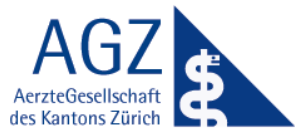

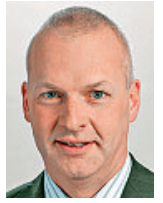

Christian Affolter

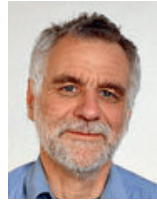

André Busato

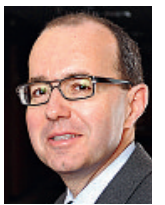

Thomas Cuen

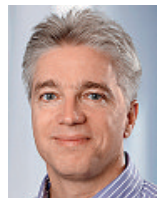

Daniel Herren

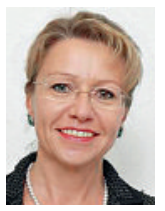

Brigitte Tag

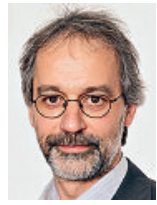

Markus ZimmermannAcklin
Der Begriff «Health Technology Assessment» (HTA) steht für die systematische Bewertung medizinischer Verfahren und Technologien. Neben den «WZW-Kriterien» (Wirksamkeit, Zweckmässigkeit, Wirtschaftlichkeit) werden dabei auch ethische, rechtliche und gesellschaftliche Aspekte berücksichtigt. HTAs sind in verschiedenen Ländern als Entscheidungshilfe bei gesundheitspolitischen Fragestellungen etabliert. Die Schweiz verfügt bisher über kein unabhängiges nationales HTA-Institut - verschiedene wichtige Akteure des Gesundheitswesens wie die FMH, die Schweizerische Akademie der Medizinischen Wissenschaften SAMW, die Schweizerische Gesundheitsdirektorenkonferenz, das Bundesamt für Gesundheit, santésuisse und die Interpharma sind jedoch in Sachen HTA aktiv geworden und engagieren sich in der Organisation «Medical Board» bzw. beim Projekt «Swiss HTA».

\section{Diskutieren Sie mit}

Können Health Technology Assessments einen wesentlichen Beitrag zur Verbesserung des Schwei-

\section{Die Podiumsgäste}

Unter der Leitung von SÄZ-Redaktorin Anna Sax, lic. oec. publ., MHA, diskutieren:

- Dr. pharm. Christian Affolter, Exec. MBA HSG, Leiter Abteilung Grundlagen santésuisse, Mitglied Project Steering Commitee Swiss HTA

- Prof. Dr. MSc André Busato, Clinical Epidemiology \& HTA, Institut für Sozial- und Präventivmedizin der Universität Bern, wissenschaftlicher Mitarbeiter Institut für Hausarztmedizin der Universität Zürich

- Thomas Cueni, Generalsekretär Interpharma Co-Chair Project Steering Commitee Swiss HTA

Die Veranstaltung wird in Zusammenarbeit mit der Ärztegesellschaft des Kantons Zürich organisiert. Die Durchführung des Anlasses wird möglich dank grosszügiger Unterstützung durch Interpharma, zer Gesundheitswesens leisten, oder wird ihr Potential überschätzt? Sind HTAs ein wichtiges Instrument einer effizienten und patientenorientierten Medizin, oder dienen sie vielmehr der Rationierung medizinischer Leistungen? Werden ärztliche Kompetenzen durch HTAs (indirekt) beschnitten, wird damit ein weiterer Schritt der Deprofessionalisierung des ärztlichen Berufsstands vollzogen? Wie bewerten die übrigen «Stakeholder» des Gesundheitswesens die Chancen und Risiken des Health Technology Assessments? $\mathrm{Zu}$ diesem Themenkreis diskutieren am nächsten Podiumsanlass der Schweizerischen Ärztezeitung profilierte Vertreter der Ärzteschaft mit Fachleuten der verschiedenen von HTA betroffenen Bereiche. Der Einbezug des Publikums in die Diskussion ist zentraler Bestandteil des Konzepts dieser Veranstaltungen, mit denen ein interessanter und konstruktiver Beitrag zur Debatte aktueller Fragen des Schweizer Gesundheitswesens geleistet werden soll.

- Dr. med. Daniel Herren, MHA, Mitglied des Zentralvorstands der FMH, Verantwortlicher Ressort Daten, Demographie und Qualität, Chefarzt Handchirurgie Schulthess Klinik Zürich, Vorstandsmitglied Trägerverein Medical Board, Mitglied Project Steering Commitee Swiss HTA

- Prof. Dr. iur. utr. Brigitte Tag, Ordinaria für Strafrecht, Strafprozessrecht und Medizinrecht, Universität Zürich, Mitglied Expertenrat Medical Board

- PD Dr. theol. Markus Zimmermann-Acklin, Lehr- und Forschungsrat (LFR), Departement Moraltheologie und Ethik, Universität Fribourg

den Verband der forschenden pharmazeutischen Industrie. Die Verantwortung für Konzept und Inhalt des Podiums liegt bei der Schweizerischen Ärztezeitung.

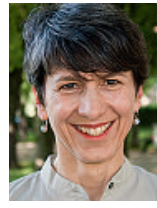

Anna Sax

\section{Eintritt frei - Anmeldung erforderlich}

Die öffentliche Podiumsdiskussion mit anschliessendem Apéro findet statt am Mittwoch, 23. November 2011, 19-21 Uhr im Hotel Marriott, Neumühlequai 42, Zürich. Der Eintritt ist frei, eine Anmeldung ist aber erforderlich. Diese kann bis Montag, 21. November via E-Mail an redaktion.saez@emh.ch oder via Fax an 0614678556 erfolgen. Bitte Ihren Namen und die Namen allfälliger Begleitpersonen sowie das Stichwort «Anmeldung zum SÄZ-Podium vom 23. November» angeben. Auch telefonische Anmeldungen sind vormittags unter 0614678572 möglich. 